Mon. Not. R. Astron. Soc. 000, 000-000 (0000) Printed 24 April $2018 \quad$ (MN LATEX style file v2.2)

\title{
Searching for giga-Jansky fast radio bursts from the Milky Way with a global array of low-cost radio receivers
}

\author{
Dan Maoz ${ }^{1}$, Abraham Loeb ${ }^{1,2}$ \\ ${ }^{1}$ School of Physics and Astronomy, Tel-Aviv University, Tel-Aviv 69978, Israel \\ ' 2 Institute for Theory and Computation, Harvard University, Cambridge, MA 02138, USA
}

24 April 2018

\begin{abstract}
If fast radio bursts (FRBs) originate from galaxies at cosmological distances, then their all-sky rate implies that the Milky Way may host an FRB every 30-1500 years, on average. If many FRBs persistently repeat for decades or more, a local giant FRB could be active now, with $1 \mathrm{GHz}$ flux radio pulses of $\sim 3 \times 10^{10} \mathrm{Jy}$, comparable to the fluxes and frequencies detectable by cellular communication devices (cell phones, Wi-Fi, GPS). We propose searching for Galactic FRBs using a global array of lowcost radio receivers. One possibility is the $\sim 1 \mathrm{GHz}$ communication channel in cellular phones, through a Citizens-Science downloadable application. Participating phones would continuously listen for and record candidate FRBs and would periodically upload information to a central data-processing website which will identify the signature of a real, globe-encompassing, FRB from an astronomical distance. Triangulation of the GPS-based pulse arrival times reported from different Earth locations will provide the FRB sky position, potentially to arcsecond accuracy. Pulse arrival times versus frequency, from reports from phones operating at diverse frequencies, or from fast signal de-dispersion by the application, will yield the dispersion measure (DM). Compared to a Galactic DM model, it will indicate the source distance within the Galaxy. A variant approach uses the built-in $\sim 100 \mathrm{MHz}$ FM-radio receivers present in cell phones for an FRB search at lower frequencies. Alternatively, numerous "software-defined radio" (SDR) devices, costing $\sim \$ 10$ US each, could be deployed and plugged into USB ports of personal computers (particularly in radio-quiet locations) to establish the global network of receivers.
\end{abstract}

Key words: stars: radio continuum, variables

\section{INTRODUCTION}

The origin and nature of fast radio bursts (FRBs) have remained enigmatic since the first FRB discovery by Lorimer et al. (2007). The 17 or so distinct FRB sources that have been reported so far are bright $(\sim 0.1-1 \mathrm{Jy})$ and brief ( $\sim 1 \mathrm{~ms}$ ) pulses of $\sim 1 \mathrm{GHz}$ radio emission (Lorimer et al. 2007; Keane et al. 2012; Thornton et al. 2013; Spitler et al. 2014; Burke-Spolaor \& Bannister 2014; Petroff et al.|2015a; Ravi et al. 2015; Champion et al. 2016; Masui et al. 2015; Keane 2016). The pulse arrival times of FRBs show a $\nu^{-2}$ frequency dependence indicative of a passage through a cold plasma, with the so-called dispersion measure (DM) measuring the line-of-sight column density of free electrons. FRBs are selected to have large measured DMs of $\sim 300-1600 \mathrm{pc} \mathrm{cm}^{-3}$, in excess of the values expected from models of the interstellar electron distribution in the Milky Way galaxy, and have therefore been inferred to originate from extragalactic sources at cosmological distances. The cosmological distance has been confirmed in the case of the sole repeating FRB 121102, which has been localised to a dwarf galaxy at redshift $z=0.19$ (Chatteriee et al. 2017; Tendulkar et al. 2017; Marcote et al. 2017).

The range of excess (above Galactic) DMs of the known FRBs correspond to a range of co-moving distances of 0.9-4.4 Gpc, with a median of $2.4 \mathrm{Gpc}$ (corresponding to a redshift of $z=0.64$ ), under the assumption that most of the excess DM is contributed by the intergalactic medium and using the standard cosmological parameters (Planck Collaboration et al. 2016). The latest estimate of the all-sky rate of FRBs with flux $>0.3 \mathrm{Jy}$ is $1.1_{-1.0}^{+3.8} \times$ $10^{4} \mathrm{day}^{-1}$ at $95 \%$ confidence (Scholz et al. 2016). This estimate is based on the single detection of FRB 121102, and ignoring the fact that it has been detected repeatedly over a period of 4 years. We note that some other recent rate estimates have smaller uncertainties, but find, to varying degrees of significance, a dependence of rate on Galactic lat- 
itude (see, e.g., Vander Wiel et al. (2016), and references therein). By adopting the Scholz et al. (2016) rate and its uncertainty, we encompass this uncertainty in the latitude dependence as well.

If FRBs occur in Milky-Way-like galaxies, we can divide the observed cosmological rate by the number of galaxies within the FRB survey volume, to find the expected FRB rate within a single such galaxy. We note that the recently localised FRB 121102 comes from an extreme-emission-line dwarf galaxy (Tendulkar et al. 2017), quite unlike the Milky Way. However, this host galaxy is not necessarily representative of all FRB hosts. The product between the comoving number density of $L_{*}$ galaxies, $\sim 10^{-2} \mathrm{Mpc}^{-3}$ (Montero-Dorta \& Prada 2009), and the cosmological volume out to the median distance of known FRBs, 57 comoving $\mathrm{Gpc}^{3}$, implies that an FRB should occur in our galaxy once per $140_{-110}^{+1400}$ years (Lingam \& Loeb 2017). A $0.3 \mathrm{Jy}$ FRB from a comoving distance of $2.4 \mathrm{Gpc}$ (a luminosity distance of $3.9 \mathrm{Gpc}$ ), placed at a typical Galactic distance of $\sim 10 \mathrm{kpc}$, would have an observed $1 \mathrm{GHz}$ flux density of $f_{\nu} \approx 3 \times 10^{10} \mathrm{Jy}$ or, equivalently, $3 \times 10^{-16} \mathrm{~W} \mathrm{~m}^{-2} \mathrm{~Hz}^{-1}$. FRB 121102 has been bursting repeatedly for at least 4 years. If most FRBs persist for decades or even centuries, a Galactic FRB could be active now. A powerful local FRB may have already been detected in the far side-lobes of radio telescope beams, but mistakenly ascribed to artificial interference.

Indeed, the radio flux density level of the received $\mathrm{GHz}$ band signals from commercial radio stations, cellular communications and wireless networks is within a few orders of magnitude of the expected flux level from a Galactic FRB. For example, a typical desktop Wi-Fi transmitter operating at $2.4 \mathrm{GHz}$ under the $802.11 \mathrm{~b}$ standard has a radiated power of $100 \mathrm{~mW}$ over an $82 \mathrm{MHz}$ bandpass with an outdoor range of $\sim 100 \mathrm{~m}$, corresponding to a detected flux density of $f_{\nu}=1 \times 10^{-14} \mathrm{~W} \mathrm{~m}{ }^{-2} \mathrm{~Hz}^{-1}$. Each of the transmitter's individual channels has a bandpass of $22 \mathrm{MHz}$, and therefore a time resolution of $\Delta t \sim 2 \times 10^{-8} \mathrm{~s}$. By binning an incoming signal into millisecond $\left(\Delta t=10^{-3} \mathrm{~s}\right)$ time bins a Wi-Fi receiver would improve its sensitivity in proportion to $\sqrt{\Delta t}$, i.e. by a factor of $\sim 200$, to a level of $f_{\nu} \sim 5 \times 10^{-17} \mathrm{~W} \mathrm{~m}^{-2} \mathrm{~Hz}^{-1}\left(5 \times 10^{9} \mathrm{Jy}\right.$, i.e. 5 GJy $)$. This is a factor 6 fainter than the typical Galactic FRB flux discussed above, and means that such a Galactic FRB, and even fainter and perhaps-more-frequent FRBs, would be detectable by existing communication devices. In the subsequent sections, we outline how an array of numerous lowcost radio receivers can be used to detect and localise giant Galactic FRBs.

\section{A GLOBAL ARRAY OF CELLULAR RECEIVERS FOR GALACTIC FRB DETECTION}

We consider below three related technical approaches to the assembly of an array of low-cost radio receivers, suitable for the detection of Galactic FRBs. The choice of the most practical approach will depend on several issues that need to be resolved, such as the ability to access and manipulate raw radio signals picked up by the antennas, the flux from FRBs at sub-GHz frequencies, the level of terrestrial noise at dif- ferent locations, and the ability to filter out that foreground noise.

\subsection{A cell phone communications channel approach}

There are currently an estimated 7 billion active cellular phone accounts on our planet (similar to the number of people), operating in several frequency bands, from 0.8 to $2.4 \mathrm{GHz}$. Each of these phones is, as argued above, a radio receiver that is in principle sensitive to a Galactic FRB signal. Furthermore, every smartphone is a programmable computer capable of analyzing the signal, of timing it up to $\Delta t \sim 10^{-7}$ s precision with its global-positioning system (GPS) module, of storing this information, and of diffusing it through the internet.

We propose therefore to build a Citizens-Science project in which participants voluntarily download onto their phones an application that runs in the background some or all of the time, monitoring the phone's antenna input for candidate broad-band millisecond-timescale pulses that appear similar to an FRB. The application would record candidate FRB pulses (most of which originate from artificial and natural noise sources) and would periodically upload the candidate pulse information (pulse profile, GPS-based arrival time), along with information about the phone (GPSbased location, operating frequency) to a central processing website. The central website will continuously correlate the incoming information from all participants, to identify the signature of a real, globe-encompassing, FRB.

Because of the received signal's integration into ms time bins (required to improve the sensitivity to FRB levels, see above), every phone's actual arrival time accuracy will be no better than $10^{-3} \mathrm{~s}$. However, improved time precision can be recovered by averaging the reported arrival times recorded by many participating phones at a similar location. For example, averaging the ms-precision reports from 10,000 phones within a city of radius $3 \mathrm{~km}$ (light travel time $<10^{-5} \mathrm{~s}$ ), would improve the precision by a factor of 100 , to $\sim 10^{-5} \mathrm{~s}$. Triangulation of the GPS-timed pulse arrival times from different Earth locations would then give the FRB sky position to an accuracy of order $\sim c \Delta t / 2 R_{\oplus} \sim 1$ arcmin. If time binning of the FRB signal, and subsequent loss of the native $10^{-7} \mathrm{~s}$ GPS timing precision could be avoided (a possibility considered in some of the other technical frameworks that we propose below), then naturally the localisation precision can be improved down to the sub-arcsecond level.

Because of the $\nu^{-2}$ arrival-time dependence of a radio pulse propagating through the Galactic plasma, phones operating at diverse frequencies (multiple networks and phone models) will receive the signal at a time delay,

$$
\delta t=0.144 \times\left(\frac{\mathrm{DM}}{200 \mathrm{pc} \mathrm{cm}^{-3}}\right)\left(\frac{\nu}{2.4 \mathrm{GHz}}\right)^{-2} \mathrm{~s} .
$$

Over, e.g., a $22 \mathrm{MHz}$ cellphone channel bandwidth at $2.4 \mathrm{GHz}$, a typical Galactic DM of $200 \mathrm{pc} \mathrm{cm}^{-3}$ (Rane \& Loeb 2016) will spread the FRB arrival time over just $2.6 \mathrm{~ms}$, comparable to typical FRB pulse widths. The channel bandwidth therefore will not result in any significant smearing of the pulse over time, which could have reduced the detection sensitivity and timing precision. By comparing the arrival times of different frequencies at the 
same locations, the central website will be able to solve for the FRB's DM that, when compared to a Galactic DM model (Cordes \& Lazio 2002; Yao et al. 2016), will indicate the FRB source distance within the Galaxy. Alternatively, the application software itself could attempt to de-disperse all candidate incoming signals across the full frequency range available to each receiver. With efficient new algorithms, real-time de-dispersion of FRB signals is now feasible on small computers (Zackav \& Ofek 2014; Zackav 2017), and so is likely possible on smartphones as well. In such a scenario, the identification of a $\nu^{-2}$ frequency sweep would be a real-time test of incoming signals, performed at the level of each individual receiver.

One clear advantage of the above operation plan is that it is essentially cost free - all of the necessary hardware (the world's cell phones) is already in place, and one needs only to carry out the plan's organizational steps in order to make it work for the scientific program. Potential problems with this proposed mode are, first, that cell phones may be hardwired at the basic electronics level to demodulate and digitize incoming communications signals, and therefore the raw broad-band radio signal containing the FRB may be inaccessible to software. Furthermore, mobile phone communications are encoded so as to allow many users to share the frequency band, and this encoding permits the detection of communication signals at sub-noise levels (as opposed to the un-encoded FRB signal).

The sought-after millisecond-timescale FRB signal will need to be disentangled from the foreground noise of cellular and other communications emissions, as well as from natural radio noise from atmospheric processes and from the sun. Although the feasibility of this requires further study, the prospects look promising based on a number of past attempts. Katz et al. (2003) review a handful of experiments, and decribe their own experiment, which is similar to our proposal. The basic concept consisted of a number of wide-angle, geographically distributed radio receivers that searched for short radio bursts, separating astronomical signals from noise by requiring coincident detections. Katz et al. (2003) used three $611 \mathrm{MHz}$ receivers in the eastern US, sensitive to $\gtrsim 3 \times 10^{4}$ Jy bursts $\left(2 \times 10^{5}\right.$ fainter than considered here) on timescales $\gtrsim 125 \mathrm{~ms}$ (50 times longer than here). The recorded, GPS-time-stamped, bursts were periodically uploaded to a central processing station, exactly as in our proposed plan.

Over 18 months of continuous operation, Katz et al. (2003) detected a burst roughly every 10 s, but $99.9 \%$ of these signals could be rejected as local interference based on their non-coincidence between the three receivers. The remaining $\sim 4000$ coincident signals could all be traced to solar radio bursts, by comparison to reports from a solar radio observatory. No other astronomical sources were detected by Katz et al. (2003) nor by previous experiments. Interestingly, Katz et al. 2003) succeeded in using their GPS signal, with its $10^{-7} \mathrm{~s}$ accuracy, to time-stamp their detected bursts to the accuracy of their $20 \mu$ s-long individual time samples, and they note that, in principle, they could have used a multiple-time-sample averaging period shorter than $0.125 \mathrm{~s}$ (at the expense of sensitivity). This would have allowed them to triangulate their source localisations, just as we propose to do.

\subsection{A cell phone FM radio channel approach}

Most or all cell phones have built-in FM-band radio receivers operating at around $\nu \sim 100 \mathrm{MHz}$ and enabling direct (i.e. not through the internet or the service provider) reception of radio broadcasts. Interestingly, this hardware is de-activated by phone manufacturers in about $\sim \frac{2}{3}$ of all phones, in the service-providers' interest of having the customers download and pay for the radio broadcasts, rather than receiving them for free. Nevertheless, about $\frac{1}{3}$ of all phones (still a sizeable number when considering the global number) do have the direct FM reception option activated. The raw, nondemodulated radio signal from this channel is more likely to be accessible to the application software in its search for an FRB signal than in the preceeding approach using the $\sim 1 \mathrm{GHz}$ cellular communication channel. A shortcoming of this option, however, is the yet-unknown properties of FRBs at $\sim 100 \mathrm{MHz}$ frequencies. Current upper limits from FRB searches at $145 \mathrm{MHz}$ (Karastergiou et al. 2015) and 139$170 \mathrm{MHz}$ (Tingay et al. 2015), limit the FRB spectral slope to $>+0.1$. As with the $\sim 1 \mathrm{GHz}$ cellular-communications option, discussed above, here too integration over time could make a typical Galactic FRB detectable at $100 \mathrm{MHz}$, even for more positive slopes as high as +1 , such that the FRB would have $\gtrsim 5$ GJy at $100 \mathrm{MHz}$. The foreground noise question in this option is similar (though in a different frequency band) to that in the previous, cellular-communications, option.

\subsection{A software-defined radio approach}

A software-defined radio (SDR) is a radio system where components such as filters, amplifiers, demodulators, etc., that are typically implemented in hardware, are implemented instead in software on a personal computer. SDR devices are widely available for $\sim \$ 10$ US a piece, and they are popular with radio amateurs. They are often the size of a memory stick and likewise can be USB plugged. An SDR device includes an antenna than can detect the full raw ambient radio emissions over some frequency range and can input them with minimal processing into a computer, where the signals can be software-processed at will. Our third approach is therefore to deploy a large number (depending on the available budget) of such SDR devices, to be plugged into participating personal computers around the globe, or base the network on devices already in use by participating radio amateurs. As with the phone option, the participants will download and install software that will continuously monitor the input from the SDR. As before, the computers will upload the information on candidate Milky Way FRBs to a central data-processing website.

A disadvantage of this approach is the need to actually buy and send the SDR hardware to the selected participating individuals of the network (unless one takes the existing-amateur-SDR approach). The advantages involve having an accessible FRB signal, uniformly processed and fully analysable at will (including spectral information from every station). Every SDR could be supplemented with a simple exterior antenna or antenna booster (wireless reception boosters are also widely and inexpensively available for cell phones and laptops) that would considerably enhance its sensitivity, lowering or fully avoiding the need for time 
integration, and hence for the sacrifice of timing precision, or simply probing for fainter and more frequent bursts (see below). Furthermore, the ability to choose the stations sites at will in a well-spaced global network, specifically in "radioquiet" locations with minimal artificial and natural radio interference, may prove to be the most important benefit.

\section{LOWER-FLUX, MORE-COMMON GALACTIC FRBS}

A major practical problem of the schemes described above are the long and uncertain timescales - decades to many centuries - expected for the detection of a single, Galactic $3 \times 10^{10}$ Jy FRB, unless typical FRBs persistently repeat for decades or centuries (which is a real possibility, given the case of FRB 121102). If FRBs typically do not repeat, then even for the more optimistic end of the rate estimate, broadcasting standards, phone models and other technical factors, may change over a decade, not to mention the limited patience of the participants and the experiment managers. A resolution of this concern, however, could be based on the fact that FRBs must have a distribution of luminosities. Indeed, if the known FRBs are at the cosmological distances indicated by their excess DMs, then they are clearly not "standard candles". A reasonable expectation is then that FRB numbers increase at decreasing luminosities. If so, lower-luminosity FRBs should be detected more frequently by the global cellular network.

Let us assume that we can parameterize the FRB number per unit luminosity with a Schechter form,

$$
\frac{d N}{d\left(\log L_{\nu}\right)} \propto L_{\nu}^{-\alpha+1} e^{-L_{\nu} / L_{F \star}}
$$

where $L_{F \star}$ corresponds to the characteristic specific luminosity of an FRB source (namely, the one that yields an observed flux density of $\sim 0.3 \mathrm{Jy}$ at a luminosity distance of $\sim 4 \mathrm{Gpc}$ ). One way to calibrate $\alpha$ is by speculating that the Galactic population of rotating radio transients (RRATs), which have some properties in common with FRBs, constitute the low-luminosity counterparts of FRBs. The rate of RRATs over the entire sky at a flux of $\sim 0.3 \mathrm{Jy}$ is $\sim 10^{6}$ day $^{-1}$, based on the estimated number of sources in the Galaxy, $\sim 10^{5}$, and their individual repetition rates, $\sim 10$ day $^{-1}$ (McLaughlin et al. 2006). The RRAT rate is thus $\sim 10^{11}$ times the Galactic FRB rate (of once per 300 yr, i.e. $10^{-5}$ day $\left.^{-1}\right)$. The RRAT flux of $\sim 0.3 \mathrm{Jy}$, in turn, corresponds to $\sim 10^{-11}$ the typical flux of a Galactic FRB. If these two populations of transient radio sources are related, then $\alpha \approx 2$. Interestingly, this value corresponds to an equal luminosity contribution from transients per logarithmic interval of luminosity.

The flux distribution from a Galactic FRB population having a particular luminosity will be $\left.\left(d N / d\left(\log f_{\nu}\right)\right)\right|_{L_{\nu}} \propto$ $f_{\nu}^{-3 / 2}$ for a spherically distributed population (e.g. in the Galactic halo), or $\propto f_{\nu}^{-1}$ for a planar distribution (e.g. the Galactic disk) - coincidentally matching the power-law scaling at low fluxes in the luminosity function for $\alpha=2$. At a 5 GJy flux level, still detectable by our proposed arrays, one might then expect to find Galactic FRBs 6 times more frequently than at 30 GJy, i.e. once per 5 to 250 years. Increasing the sensitivity by one or two orders of magnitudes, e.g. by adding simple antennas in the SRD option, would potentially allow for the detection of Galactic FRBs on a yearly to weekly basis, and for the direct determination of their luminosity function.

\section{CONCLUSIONS}

The first, and so-far only, FRB that has been localised, FRB 121102, is at a cosmological distance, it has been repeating for at least 4 years, and its host galaxy is a lowmetallicity dwarf. We have argued that if most FRBs are cosmological, but their hosts are not necessarily dwarf galaxies like the host of FRB 121102, then their all-sky rate implies that the Milky Way hosts an FRB every 30 to 1500 years. If, furthermore, many FRBs repeat like FRB 121102, and for long enough, then the occurrence frequency could be higher, and a local FRB may even be active now. A typical Galactic FRB will be a millisecond broad-band radio pulse with $1 \mathrm{GHz}$ flux density of $\sim 3 \times 10^{10} \mathrm{Jy}$, not much different from the radio flux levels and frequencies detectable by cellular communication devices (cell phones, WiFi, GPS). If the Milky Way has a currently active and repeating FRB source, then some Local Group galaxies would have them too, at MJy flux-density levels, which could be detected by monitoring nearby galaxies with dedicated small radio telescopes.

An argument against frequent Galactic FRBs could be that FRBs require some kind of exotic and energetic physical event, such as a super-flare from a magnetar, and that irradiation of the Earth by such an event once per century or millenium would be accompanied by clear signatures, or perhaps even by mass extinctions. However, this argument relies on a still-speculative connection between the radio emission of FRBs and their emissions in other bands. Observationally, an upper limit of $10^{8} \mathrm{Jy}$ has been set on any FRB-like radio flux accompanying the giant 2004 December $\gamma$-ray burst from the magnetar SGR 1806-20, and no $\gamma$-ray counterparts have been detected for any FRB (Tendulkar et al. 2016).

Our proposed search for Galactic FRBs using a global array of low-cost (possibly already existing) radio receivers would enable triangulation of the GPS-timed pulse arrival times from different Earth locations, localising the FRB sky position to arcminute or even arcsecond precision. Pulse arrival times from devices operating at diverse frequencies, or from de-dispersion calculations on the devices themselves, will yield the DM that, when compared to a Galactic DM model, will indicate the FRB source distance within the Galaxy. Fainter FRBs could potentially be detected on a yearly or even weekly basis, enabling a direct measurement of the FRB luminosity function.

\section{ACKNOWLEDGMENTS}

We thank C. Carlsson, A. Fialkov, J. Guillochon, Z. Manchester, E. Ofek, M. Reid, B. Zackay, and the anonymous referee, for useful advice and comments. This work was supported in part by Grant 1829/12 of the I-CORE program of the PBC and the Israel Science Foundation (D.M.) and by a grant from the Breakthrough Prize Foundation (A.L.). 
A.L. acknowledges support from the Sackler Professorship by Special Appointment at Tel Aviv University.

\section{REFERENCES}

Burke-Spolaor, S., \& Bannister, K. W. 2014, ApJ, 792, 19

Chatterjee, S., et al. 2017, Nature, 541, 58

Champion, D.J., Petroff, E., Kramer, M., et al. 2016, MNRAS, 460, L30

Cordes, J. M., \& Lazio, T. J. W. 2002, arXiv:astro-ph/0207156

Karastergiou, A., Chennamangalam, J., Armour, W., et al. 2015, MNRAS, 452, 1254

Katz, C.A., Hewitt, J.N., Corey, B.E., Moore, C.B 2003, PASP, 115, 675

Keane, E. F., Stappers, B. W., Kramer, M., \& Lyne, A. G. 2012, MNRAS, 425, L71

Keane, E. F. 2016, MNRAS, 459, 1360

Lingam, M. \& Loeb, A. 2017, arXiv:1701.01109

Lorimer, D. R., Bailes, M., McLaughlin, M. A., Narkevic, D. J., \& Crawford, F. 2007, Science, 318, 777

Marcote, B., et al. 2017, ApJ, 834, L8

Masui, K., Lin, H.-H., Sievers, J., et al. 2015, Nature, 528, 523

McLaughlin, M. A., Lyne, A. G., Lorimer, D. R., et al. 2006, Nature, 439, 817

Montero-Dorta, A. D., \& Prada, F. 2009, MNRAS, 399, 1106

Petroff, E., Bailes, M., Barr, E. D., et al. 2015, MNRAS, 447, 246

Planck Collaboration, Ade, P. A. R., Aghanim, N., et al. 2016, A\&A, 594, A13

Rane, A., Loeb, A. 2016, arXiv:1608.06952

Ravi, V., Shannon, R. M., \& Jameson, A. 2015, ApJ, 799, L5

Scholz, P., Spitler, L. G., Hessels, J.W. T. et al. 2016, ApJ, 833, 177

Spitler, L. G., Cordes, J. M., Hessels, J. W. T., et al. 2014, ApJ, 790, 101

Tendulkar, S.P., Kaspi, V.M., \& Patel, C. 2016, ApJ, 827, 59

Tendulkar, S.P., et al. 2017, ApJ, 834, L7

Tingay, S. J., Trott, C. M., Wayth, R. B., et al. 2015, AJ, 150, 199

Thornton, D., Stappers, B., Bailes, M., et al. 2013, Science, 341,53

Vander Wiel, S., Burke-Spolaor, S., Lawrence, E., Law, C.J., Bower, G.C. 2016, arXiv:1612.00896

Yao, J. M., Manchester, R. N., \& Wang, N. 2016, arXiv:1610.09448

Zackay, B., Ofek, E. 2014, arXiv:1411.5373

Zackay, B. 2017, American Astronomical Society Meeting Abstracts, 229, 330.06 